\title{
Formation, structure and promoting crystallization capacity of stereocomplex crystallite network in the poly(lactide) blends based on linear PLLA and PDLA with different structures
}

Zhanxin Jing, Xutao Shi ${ }^{*}$, Guangcheng Zhang ${ }^{*}$, Jiang Li, Jianwei Li, Lisheng Zhou, Hongming Zhang

MOE Key Lab of Applied Physics and Chemistry in Space, College of Science, Northwestern Polytechnical University, Xi'an, 710072, China

\section{ABSTRACT}

A series of asymmetric PLLA/PDLA blends based on linear PLLA and PDLA (linear PDLA and star-shaped PDLA) were prepared by solution blending, and stereocomplex crystallites (sc-crystallites) were formed between PLLA and various PDLA due to strong hydrogen bonding. Rheological results indicated that the melt strength of PLLA was improved due to the network structure of sc-crystallites in the PLLA melt. The effect of PDLA on rheological behavior of the melt decreased in the following order: 6PDLA>L-PDLA>3PDLA>4PDLA. Dissolution experiment revealed that the formed network structure could be formed by interparticle polymer chains or branched points. Nonisothermal and isothermal crystallization showed that the promoting crystallization of sc-crystallites for PLLA was closely related to thermal treatment temperature, crystallization temperature and the structure of PDLA,

\footnotetext{
* Correspondence to: Xuetao Shi (shixuetao@nwpu.edu.cn) and Guangcheng Zhang (zhangguc@nwpu.edu.cn),Department of Applied Chemistry, Northwestern Polytechnical University, Xi' an, 710072, China.
} 
and the PDLA structure could change the pattern of crystal growth during isothermal crystallization. Nucleation efficiency of sc-crystallites during non-isothermal crystallization decreased with increasing of arm numbers of PDLA. POM results also demonstrated that the nucleation and spherulite growth during isothermal crystallization were affected by the PDLA structure. This study has systemically investigated the effects of the PDLA structure on rheology and crystallization capacity of asymmetric PLLA/PDLA blends, which would provide potential approaches to control the microstructure and physical performances of PLLA/PDLA blends.

Keywords: stereocomplex; crystallization; rheology; network structure

\section{Introduction}

Poly(lactide) (PLA), which can be derived from nature resources (such as corn starch, sugar beets), is a green and sustainable development plastic with the good biocompatibility and biodegradability $[1,2]$. Most importantly, the thermal processing performance and mechanical properties of PLA are similar with polypropylene (PP), polyethylene (PE) and other common plastics [3]. Now it has attracted much attention from researchers due to its broad application prospects and huge market potential. However, PLA also has significant drawbacks, such as brittleness (fracture elongation rate), poor thermal stability, slow crystallization rate and low melt strength, which have greatly limited the application of PLLA as an engineering plastic [4-8]. The slow crystallization rate and poor melt strength of PLA are major industrial problems. Nowadays many efforts [9-13] have been done to improve the performance of PLA. The nucleation and crystallization rates of PLA could be improved easily by adding a 
nucleating agent. The incorporation of heterogeneous nucleation sites into the polymer matrix could obviously decrease the energy barrier for polymer crystallization, which would promote the crystallization kinetics substantially [5]. High melt strength of polymer can be acquired by introducing branch structure or preparing polymer with high molecular weight [8]. Therefore, it is a great challenge to found a new method that it could prepare PLA with fast crystallization rate and high melt strength at the same time.

Poly(lactide) stereocomplex (sc-PLA) have been widely investigated due to its excellent performance since it was firstly reported by Ikada in 1987 [14]. The previous literatures have shown that sc-PLA can be formed by the strong hydrogen bonding between PLLA and its enantiomer PDLA, and its physical properties are different from either PLLA or PDLA $[15,16]$. The melting point of sc-crystallites is about $50^{\circ} \mathrm{C}$ higher than that of homocrystallites [17,18]. And stereocomplex formation between PLLA and poly(D-lactide) can enhance the mechanical properties and hydrolytic/thermal degradation resistance of PLA-based materials [16,19]. This is because that the crystallite of sc-PLA is $\beta$ crystal with $3_{1}$ helical conformation in a trigonal cell, which is different from $\alpha$ crystal of homopolymer with $10_{3}$ helical conformation [20]. Now sc-PLA has been used in many applications, such as nucleating agent and rheological modifier. Yamane et al [21] and Tsuji et al [24] have reported that the addition of small amounts of PDLA is effective to accelerate PLLA crystallization. Ma and Wei [22,23] found that sc-crystallites reserved at the melt of PLLA could form network structure, resulting in a transition from the liquid-like to 
solid-like viscoelastic behavior. Many factors, such as the concentration of PDLA, molecular weight, optical purity, fine structures of sc-crystallites, initial melt states, preparation method and crystallization conditions, have been analyzed to discuss the effects of sc-crystallites on the properties of PLLA [21-31].

For asymmetric PLLA/PDLA blends, the common investigation focuses on the system of linear PLLA and linear PDLA. And the compared study of asymmetric linear-/star-shaped poly(lactide) blends is rarely reported. Especially, the structure of sc-crystallites in the asymmetric linear-/star-shaped poly(lactide) blends and its effect on the polymer melt and crystallization capacity have not been reported by any researcher. Therefore, the investigation of asymmetric PLLA/PDLA blends with different molecular structures of PDLA in the field has a certain theoretical significance and guiding significance for engineering application. In this study, a simple method was used to improve melt strength and crystallization capacity of PLLA through solution blending with 5.0wt\% of linear or star-shaped (different arm numbers) PDLA. PDLA concentration was so low that stereocomplex could not either be a continuous phase or connect all PLLA chains as cross-linking points. The existence of this structure was investigated by a rheological approach and dissolution experiment. The effect of this structure on PLLA crystallization was in-depth discussed.

\section{Experimental}

\subsection{Materials and reagents}

PLLA $\left(M_{w}=2.0 \times 10^{5} \mathrm{~g} / \mathrm{moL}\right)$ was purchased from American Nature Works 
Company. The catalyst $\mathrm{Sn}(\mathrm{Oct})_{2}$ and initiators (1-Dodecanol, 1,1,1-Trimethylolpropane Pentaerythrotol and Inositol) were obtained from J\&K Chemicals Co. Ltd. Other reagents were analytical grade without further purification.

\subsection{Synthesis and characterization of PDLA with different structures}

Poly(D-lactide) with different structures were synthesized by ring-opening polymerization in the presence of different initiator. Firstly, D-lactide, initiator and catalyst $\mathrm{Sn}(\mathrm{Oct})_{2}(1.0 \mathrm{wt} \%$, related to the weight of D-lactide) were added to a tube. The tube was purged with nitrogen to eliminate air, and pumped vacuum until the degree of vacuum below $30 \mathrm{~Pa}$. Then the tube was sealed and placed in an oil bath of $140^{\circ} \mathrm{C}$ for $72 \mathrm{~h}$. After the reaction, the obtained substance was dissolved in chloroform, and precipitated using an excess of methanol. The obtained precipitate was filtered under decompression. The purification process was repeated three times to remove completely the catalyst $\mathrm{Sn}(\mathrm{Oct})_{2}$ and the unreacted monomer D-lactide. Finally, the obtained substance was dried at a vacuum oven for $48 \mathrm{~h}$ at $60^{\circ} \mathrm{C}$. The obtained linear PDLA was marked as L-PDLA. The prepared star-shaped PDLA (S-PDLA) was marked as X PDLA, where $\mathrm{X}$ represents arm number of star-shaped polymer. The synthetic PDLA with different structures were characterized by NMR (300 MHz, Brucker AM300 FT, $\mathrm{CDCl}_{3}$ solvent), GPC (Waters 1515, tetrahydrofuran eluent) and DSC (Mettler DSC1), and the results are shown in Figs.S1 and S2 and Table1.

\subsection{Preparation of PLLA/PDLA blends}

PLLA and PDLA were dissolved into dichloromethane solution, respectively. 
Then two solutions were fully mixed by a magnetic stirrer. The obtained solution was poured into the polytetrafluoroethylene mold for two days under room temperature for evaporation of the solvent dichloromethane. Afterwards, the obtained blend films were dried in a vacuum oven for $24 \mathrm{~h}$ at $50{ }^{\circ} \mathrm{C}$ to eliminate the residual solvent. The $\mathrm{D} / \mathrm{L}$ weight ratio of the prepared blend films were fixed at 5:95.

\subsection{Characterization of blends}

Fourier transform infrared spectra of blends were measured with FT-IR instrument using $\mathrm{KBr}$ disc as background in the range of 4000 to $500 \mathrm{~cm}^{-1}$ at the resolution of $4 \mathrm{~cm}^{-1}$.

XRD curves of blend films were obtained by a Brucker X-ray diffractometer, using $\mathrm{Cu} \mathrm{Ka}$ radiation source in the scanning angle range of $2 \theta=5^{\circ}-35^{\circ}$ at the scanning rate of $4 \% \mathrm{~min}$.

Rheological behavior of PLLA/PDLA blends was investigated using a DHR-2 rheometer (TA instruments, USA) with a parallel plate of $25 \mathrm{~mm}$ in diameter. Samples were first melted and prepared to be films of $1.0 \mathrm{~mm} \times 25 \mathrm{~mm}$ (thickness $\times$ diameter). The obtained films were placed to the rheometer and then kept for 4 min at $190^{\circ} \mathrm{C}$. Afterwards samples were measured from 0.1 to $500 \mathrm{rad} / \mathrm{s}$ with a strain of $1.0 \%$.

Dynamic light scattering measurements were performed onto a particle analyzer to analyze particle sizes of stereocomplex in the PLLA/PDLA suspensions. The suspensions were prepared by dissolving of PLLA/PDLA film into dichloromethane, and the suspension concentration was about $0.1 \mathrm{mg} / \mathrm{mL}$.

Melting and crystallization behavior Thermal analysis was measured by a 
METLLER differential scanning calorimetry (DSC) under nitrogen protection. Melting behavior of blends was performed by DSC from $20^{\circ} \mathrm{C}$ to $240^{\circ} \mathrm{C}$ at $10^{\circ} \mathrm{C} / \mathrm{min}$. For the non-isotherm crystallization behavior, samples were first heated to $190^{\circ} \mathrm{C}$ at $50^{\circ} \mathrm{C} / \mathrm{min}$, and held at this temperature for $4 \mathrm{~min}$ to erase thermal history. Then samples were cooled to $20^{\circ} \mathrm{C}$ at $2^{\circ} \mathrm{C} / \mathrm{min}$, and reheated to $190^{\circ} \mathrm{C}$ at $10^{\circ} \mathrm{C} / \mathrm{min}$. The effect of thermal treatment temperature on crystallization behavior of the blends was examined by the following method: sample was cooled to $80^{\circ} \mathrm{C}$ at $2^{\circ} \mathrm{C} / \mathrm{min}$ after melting at the certain temperature $\left(180^{\circ} \mathrm{C}, 190^{\circ} \mathrm{C}, 200^{\circ} \mathrm{C}, 210^{\circ} \mathrm{C}, 220^{\circ} \mathrm{C}\right.$ and $\left.230^{\circ} \mathrm{C}\right)$ for $4 \mathrm{~min}$, and reheated to $190^{\circ} \mathrm{C}$ at $10^{\circ} \mathrm{C} / \mathrm{min}$. For investigating isothermal crystallization, the sample was fast cooled from $190^{\circ} \mathrm{C}$ to the desired temperatures $\left(110^{\circ} \mathrm{C}, 120^{\circ} \mathrm{C}, 125^{\circ} \mathrm{C}, 130^{\circ} \mathrm{C}, 135^{\circ} \mathrm{C}\right.$ and $\left.140^{\circ} \mathrm{C}\right)$ at $50^{\circ} \mathrm{C} / \mathrm{min}$ and then held for enough time at this temperature for crystallization, and followed by reheated to $190^{\circ} \mathrm{C}$ at $10^{\circ} \mathrm{C} / \mathrm{min}$ to examine the melting behavior.

Nucleation efficiency evaluation of sc-crystallites In order to investigate the nucleation efficiency of sc-crystallites in the asymmetric PLLA/PDLA blends, the self-nucleation procedure of the neat PLLA was measured by DSC. The special procedures were as follows [23,32]: Firstly, the neat PLLA was heated to $190^{\circ} \mathrm{C}$ to erase the heat history and kept for 4 min at the temperature. Then sample was cooled to $80^{\circ} \mathrm{C}$ at the rate of $2.0^{\circ} \mathrm{C} / \mathrm{min}$, which is similar with the cooling rate in the non-isothermal crystallization. This offers a standard heat history; Secondly, the neat PLLA was heated to the certain temperature (self-nucleation temperature) in the partial melting zone and held at the temperature for 4 min to produce self-nucleated 
seeds, and then sample was cooled to $80^{\circ} \mathrm{C}$ at $2^{\circ} \mathrm{C} / \mathrm{min}$. The crystallization temperatures $\left(T_{\mathrm{c}}\right)$ of self-nucleated PLLA were obtained from the cooling curves. Thirdly, DSC heating curves of samples after melted at self-nucleation temperature for $4 \mathrm{~min}$ were also measured. Samples were heated to $190^{\circ} \mathrm{C}$ at $10^{\circ} \mathrm{C} / \mathrm{min}$ to observe whether the crystallites were completely melted at self-nucleation temperature.

Spherulite morphology The nucleation and crystalline morphology of samples at the isothermal crystallization temperature of $130^{\circ} \mathrm{C}$ were observed using a polarizing optical microscopy (POM, Olympus CO., Tokyo, Japan) equipped with a hot-stage (LINKAM THMS600). Samples were first placed at the glass slide, and kept at $190^{\circ} \mathrm{C}$ for $4 \mathrm{~min}$. Then sample was quickly cooled to $130^{\circ} \mathrm{C}$ at the rate of $50^{\circ} \mathrm{C} / \mathrm{min}$ to observe the nucleation and spherulite morphology.

\section{Results and Discussion}

\subsection{Stereocomplex formation}

Fig. 1(a) shows the finger-print region of $1000-850 \mathrm{~cm}^{-1}$ at FT-IR spectra of PLLA and PLLA/PDLA blends. The neat PLLA shows an obvious peak at $921 \mathrm{~cm}^{-1}$, assigned to the homocrystallites of PLLA. With addition of PDLA into the PLLA matrix, a novel peak at FT-IR spectra of asymmetric PLLA/PDLA blends is observed at $908 \mathrm{~cm}^{-1}$, assigned to sc-crystallites in the asymmetric PLLA/PDLA blends $[15,33]$. DSC curves of PLLA and PLLA/PDLA blends are displayed in Fig. 1(b). For the neat PLLA, there is only one melting peak at about $168^{\circ} \mathrm{C}$. However, PLLA/PDLA blends show two melting peaks at about $168^{\circ} \mathrm{C}$ and $217^{\circ} \mathrm{C}$, which are attributed to homocrystallites and stereocomplex crystallites, respectively [35]. Fig. 1(c) displays 
XRD patterns of PLLA and PLLA/PDLA blends. The neat PLLA shows several diffraction peaks at $14.8^{\circ}, 16.6^{\circ}$, and $19^{\circ}$, assigned to $\alpha$ crystal of homocrystallites $[34,35]$. For XRD curves of blends, there are the new diffraction peaks at $11.9^{\circ}$ and $20.6^{\circ}$, assigned to the characteristic peaks of sc-crystallites [36]. These results indicate that stereocomplex crystallites are formed in the blends based linear PLLA and PDLA with different structures.

\subsection{Rheological behaviors of PLLA/PDLA blends}

The rheological behavior of the neat PLLA and PLLA/PDLA blends at the temperature of $190^{\circ} \mathrm{C}$ were explored by an oscillatory shear rheometer. And the obtained storage modulus $\left(\mathrm{G}^{\prime}\right)$, loss modulus $\left(\mathrm{G}^{\prime \prime}\right)$, loss tangent $(\tan \delta)$, and complex viscosity $\left(\eta^{*}\right)$ of the neat PLLA and PLLA/PDLA blends as function of frequency are displayed in Fig. 2. Fig. 2(a) and (b) show the storage modulus $\left(\mathrm{G}^{\prime}\right)$ and loss modulus $\left(\mathrm{G}^{\prime \prime}\right)$ of the neat PLLA and PLLA/PDLA blends as function of frequency, respectively. It is obvious that the storage modulus and loss modulus increase as the frequency increases from 0.1 to $500 \mathrm{rad} / \mathrm{s}$, and the change of storage modulus is more significant than that of loss modulus. For the neat PLLA, it shows the typical terminal behavior with the scaling law of approximate $\mathrm{G}^{\prime} \propto \omega^{2}$ and $\mathrm{G}^{\prime \prime} \propto \omega$. When PDLA is incorporated into PLLA matrix, the storage modulus and loss modulus rapidly increases with respect to that of the neat PLLA. The PLLA/PDLA blends display an obvious solid-like behavior at the low frequency zone. This reveals that a slow relaxation process and an increased elasticity of the blend melt $[37,38]$. This may be attributed to the sc-crystallites reserved in the melt of PLLA/PDLA blends, which could play a role 
of cross-linking agents. Similar results have been reported by Ma and Wei [22,23]. And the change of the storage modulus and loss modulus of the PLLA/PDLA blends is closely related to the structure of PDLA in the asymmetric PLLA/PDLA blends. At low frequency zone, the storage modulus of the blends decreases in the following order: PLLA/6PDLA>PLLA/L-PDLA>PLLA/3PDLA>PLLA/4PDLA, while the loss modulus of the blends follows the order: PLLA/6PDLA > PLLA/L-PDLA>PLLA/4PDLA >PLLA/3PDLA.

Loss tangent $(\tan \delta)$, which is the ratio of loss modulus and storage modulus, is a critical parameter characterizing the relaxation behavior of the viscoelastic materials. It is more sensitive to the relaxation changes than $G^{\prime}$ and $G^{\prime \prime}$ [39]. To further investigate the effect of the structure of PDLA in the PLLA/PDLA blends on rheological behavior of the blends, loss tangent of the neat PLLA and PLLA/PDLA were discussed, as shown in Fig. 2(c). The neat PLLA rapidly decreases with increasing of the frequency, which is a typical behavior of viscoelastic liquid. Loss tangent decreases obviously compared to that of neat PLLA when PDLA is added into PLLA matrix. And a broad peak is observed for PLLA/PDLA blends. This indicates that there exists an elastic response, which may be attributed to the network structure in the melt of PLLA/PDLA blends due to the cross-linking effect of sc-crystallites [33]. It is also clear that the values of $\tan \delta$ decreases in the following order: PLLA >PLLA/4PDLA >PLLA/3PDLA >PLLA/L-PDLA >PLLA/6PDLA, revealing that the cross-linking density of the blends increases in this order $[8,40]$. This indicates that the structure of PDLA affected directly the elastic response of 
PLLA/PDLA blends. This may be attributed to the difference of network structure formed PLLA and PDLA with different structures.

Fig. 2(d) displays frequency dependence of complex viscosity $\left(\eta^{*}\right)$ of the neat PLLA and PLLA/PDLA blends at the temperature of $190^{\circ} \mathrm{C}$. The neat PLLA showed the Newtonian plateau at low frequency $(\omega<10 \mathrm{rad} / \mathrm{s})$. At high frequency $(\omega>10 \mathrm{rad} / \mathrm{s})$, complex viscosity decreases with increasing frequency, which is attributed to shear thinning. In contrast, the complex viscosity of PLLA/PDLA blends has steep slopes. No-Newtonian plateau is observed at all the range of frequency. This indicates that sc-crystallites obviously reinforce the PLLA melt. It can be found that the complex viscosity of the melt of blends at lower frequency becomes lower in the following order: PLLA/6PDLA>PLLA/L-PDLA>PLLA/3PDLA>PLLA/4PDLA. This reveals that the formed sc-crystallites have the obvious difference for reinforcing the melt due to the different of the structure of PDLA.

The above results have revealed that the incorporation of PDLA into the PLLA matrix obviously reinforces the melt of PLLA due to the formed network structure by the cross-linking effect of sc-crystallite. Usually, crystallites possess a high modulus, so the dispersed crystallites in the melt could reinforce the melt $[41,42]$. But the part of PLLA chains is incorporated into sc-crystallites, which act as the core surrounded by PLLA chains. Therefore, sc-crystallites not only increase the molecular weight, but also play a role of cross-linking point. So this structure could greatly improve the melt strength of polymer. The above findings also demonstrate that the difference of rheological behavior of the blend melt is closely related to the structure of PDLA and 
the formation mechanism of sc-crystallite. For linear PLLA and linear PDLA, sc-crystallites would be formed by layer by layer arrangement of L-segment and D-segment $[43,44]$. In the asymmetric PLLA/PDLA blends, the larger the sc-crystallites formed, the more the incorporation of PLLA chains in sc-crystallites. For linear PLLA and star-shaped PDLA, linear L-segment would form sc-crystallites with each arm of S-PDLA by strong hydrogen bonding [3]. So the formation of sc-crystallites in the asymmetric PLLA/S-PDLA blends is very complicated. Compared to the blend PLLA/L-PDLA, the blends PLLA/S-PDLA contains the branch points due to the branch structure of S-PDLA. While the arm length of PDLA with similar molecular weight decreases with increasing of arm number, resulting in small sc-crystallites. This would decrease the effect of crystallite for reinforcing polymer melt, but the reserved sc-crystallites in the PLLA/S-PDLA blends may form more cross-linking points. Therefore, the structure of formed network in the asymmetric PLLA/S-PDLA is very complicated with respect to that of PLLA/L-PDLA.

\subsection{Structure of the formed stereocomplex network}

Rheological experiments have demonstrated that there exhibits a network structure at the melt of asymmetric PLLA/PDLA blends due to the special structure of sc-crystallite related to the branched structure of star-shaped PDLA. However, the investigation on the structure of formed network is not reported. Horst et al [45] and Coppola et al [46] found that there formed analogous cross-linking structure during crystallization process. For linear polymer, there exist three possible structures: (i) 
impingement of amorphous chains, immobilized by their segment attachment within a crystalline structure, with similarly immobilized chains from adjacent structures; (ii) a network of bridging molecules which have segments in neighboring sc-crystallites; (iii) immediate contact between sc-crystallites. As shown in Fig. 3(a), the structures of A-C, D, and E stand for the probabilities of (i), (ii) and (iii), respectively. With the structure evolution from A to $\mathrm{E}$, the distance between neighboring sc-crystallites should decrease. It is known that the formation mechanism of stereocomplex between linear PLLA and star-shaped PDLA is different from linear PLLA and linear PDLA. Shao et al [3] have reported that the branch of 3PDLA and PLLA chain in the PLLA/3PDLA were packed side by side orderly to sc-crystallites. So the branched structure is retained completely in the blends. As shown in Fig. 3(b), there exists several possible when linear PLLA and star-shaped PDLA forms sc-crystallites: (i) a branch of S-PDLA only participates into the formation of sc-crystallites; (ii) all braches joins into sc-crystallite formation; (iii) the part of the branches $(\geq 2)$ is embedded into sc-crystallites. Therefore, for asymmetric PLLA/S-PDLA blends, it could contain the structure described in Fig.3(b), which would enable the formed network structure more complicated. Especially for the structure of b, c, d and e in Fig. 3(b), the cross-linking points of sc-crystals is obviously improved due to the branched structure of S-PDLA. Therefore, the network structure in asymmetric PLLA/S-PDLA blends could be formed by cross-linking effect of sc-crystallites and the branched structure of S-PDLA. However, S-PDLA possesses larger steric hindrance, which may decreases the arm numbers of S-PDLA embedding into sc-crystallites. 
To investigate the structure of the formed network, the dissolution experiment of PLLA/PDLA blends were measured using dichloromethane as the solvent. It is known that the dichloromethane is a good solvent for PLLA, but it could not dissolve sc-crystallites. From the dissolution experiment, the sc-crystallites were not connected together as a whole but were separated from each other and suspended homogeneously in the dichoromethane solution. Therefore, the structures of D and E in PLLA/L-PDLA blend could be excluded. Because neighboring sc-crystallites are immediately connected by each other and by the bridging molecules, sc-crystallites are not dissolved and still connected by each other by these bridging molecules which have segments in neighboring sc-crystallites after the uncomplexed PLLA and PDLA chains are dissolved into dichoromethane [22, 46]. For the cases of A-C, neighboring sc-crystallites are connected by the amorphous PLLA chains, which can be dissolved in dichoromethane. So the neighboring sc-crystallites will be separated with each other in the solvent after these amorphous chains dissolved in dichoromethane $[22,46,47]$. Therefore, the structures of A-C may be coexist in the asymmetric PLLA/L-PDLA blends. For PLLA/S-PDLA blends, the network structure would be formed by the structures of A-C in Fig.3(a) and the branched structures described in Fig.3(b) The obtained size distribution of sc-network structure is displayed in Fig. 4. It is clear that there exist colloidal particles in the dichloromethane solution of PLLA/PDLA, and the size distribution of colloidal particles presents the multimodal distribution. Narital et al [48] found that the lamellar thickness of sc-crystallites in the asymmetric PLLA/PDLA blends is about 20nm. As shown in Fig. 3(b), the 
amorphous segments and branched structure produces the network structure, results in the agglomerate of sc-crystallites. In Figure 3, there is a peak at about 20nm except for the blend PLLA/4PDLA, which may be attributed to the lamellar of sc-crystallites that could not participate into the agglomerate. This may be attributed to the steric hindrance of S-PDLA, which decreases the arm numbers of S-PDLA embedding into sc-crystallites. With increasing of the arm numbers of PDLA participated into sc-crystallites, the larger colloid presents more distribution peaks of colloid, as shown in Fig.4(d). The increased arm numbers offers more chance for the formation of colloid with different particle size.

\subsection{Non-isothermal crystallization behaviors of PLLA/PDLA blends}

Fig. 5(a) and (b) show the DSC cooling curves and heating curves of PLLA and PLLA/PDLA blends cooling from $190^{\circ} \mathrm{C}$, and the obtained thermal parameters are listed in Table 2. In Fig. 5(a), the crystallization peak of the neat PLLA appears at $100.9^{\circ} \mathrm{C}$. For the blends, their crystallization temperature decreases from $121.6^{\circ} \mathrm{C}$ to $96.7^{\circ} \mathrm{C}$ with increasing of the arm number of PDLA, indicating that the reserved sc-crystallites in asymmetric PLLA/PDLA blends presents different promoting capacity of PLLA crystallization. This may be attributed to the PDLA structure. The dissolution experiment has demonstrated that the PDLA structure impacts directly on the structure of sc-crystallite network, and the rheology results indicate that cross-linking density of sc-crystallite network in the PLLA/PDLA is affected by the PDLA structure. Therefore, the PDLA structure is critical factor affecting the crystallization of PLLA/PDLA blends. Except the neat PLLA, the cold crystallization 
peak of the blends decreases or disappears with respect to the neat PLLA in Fig. 5(b). Furthermore, the blends show a sharp melting peak instead of the typical double melting peaks. This indicates that PDLA obviously accelerates the crystallization of PLLA. It is clear form Table 2 that crystallinity of PLLA and the blends decreases in the following order: PLLA/4PDLA>PLLA/L-PDLA>PLLA/6PDLA >PLLA/3PDLA>PLLA. In this study, PLLA/PDLA blends are prepared by solution blending, which would make the fully stretch of polymer segment. So all PDLA in the blends participate into the sc-crystallites formation. Thermal treatment temperature of $190^{\circ} \mathrm{C}$ is lower than the melting temperature of sc-crystallites $\left(T_{m, s c}\right)$, which could act as nucleation sites and promote the PLLA crystallization. For PLLA/L-PDLA blends, the concentration of PDLA in the blends directly affects the PLLA crystallization. In our study, the blends contain the similar concentration of S-PDLA, but they show different crystallization behavior. This may be attributed to the chemical structure of PDLA. The LDS results of Fig. 4 have demonstrated that the PDLA structure affects the agglomerate of sc-crystallites in the PLLA/PDLA blends. It is known that the particles with either smaller or larger surface area could not play a role of heterogeneous nucleation agent [21]. So the PDLA structure affects directly the nucleation efficiency of sc-crystallites. The cross-linking effect of sc-crystallites also limits the crystallization of PLLA. Therefore, the crystallization capacity of the blends is a complicated problem, which is closely related to the nucleation and the cross-linking effect of sc-crystallites.

The effect of thermal treatment temperature on crystallization behavior of the 
blends PLLA/L-PDLA and PLLA/6PDLA was also investigated, and the obtained results are shown in Fig. 6. Fig. 6(a) shows DSC cooling curves of PLLA/L-PDLA after melting at different temperatures. The PLLA crystallization temperatures of the blends melted at $180-200^{\circ} \mathrm{C}$ are higher than those of the blends melted at $210 \sim 230^{\circ} \mathrm{C}$. Fig. 6(b) displays DSC heating curves of PLLA/L-PDLA. The melting peak of PLLA/L-PDLA treated at the range of $180 \sim 200^{\circ} \mathrm{C}$ doesn't have the obvious change, while melting peak becomes width and the melting temperature shifts to lower temperature when thermal treatment temperature exceeds $210^{\circ} \mathrm{C}$. And DSC curves present the obvious exothermic peak when the treating temperature is 220 or $230^{\circ} \mathrm{C}$, revealing the decreasing of promoting crystallization capacity of sc-crystallites. This is attributed to thermal stability of sc-crystallites. The melting temperature of sc-crystallites in the blends is about $210 \sim 220^{\circ} \mathrm{C}$ as shown in Fig.1(b). At lower thermal treatment temperature $\left(<T_{m, s c}\right)$, sc-crystallites reserved in the blends plays a role of heterogeneous nucleation sites, accelerating the crystallization of PLLA. At higher treatment temperature $\left(>T_{m, s c}\right)$, sc-crystallites are completely destroyed. Although sc-crystallites could be formed again during the cooling process, its number and size is obviously different. As shown in Fig.6(a) and Fig.6(b), the promoting crystallization capacity of re-formed sc-crystallites is lower than the reserved sc-crystallites in the PLLA/L-PDLA blends. Fig. 6(c) and Fig 6(d) displays DSC cooling curves of PLLA/6PDLA after treated at different temperatures and the subsequent heating curves. In Fig.6(c), the crystallization temperature of the blends first decreases and then increases with increasing of thermal treatment temperature, 
which is different with the PLLA/L-PDLA blends. It is clear that cold crystallization peak appears when thermal treatment temperature is $230^{\circ} \mathrm{C}$. This is related closely to the PDLA structure. Sc-crystallites based on PLLA and 6PDLA exist more defect compared to PLLA and L-PDLA because of branched structure and steric hindrance. With increasing of thermal treatment temperature, the imperfect sc-crystallites are first melted and the size of perfect sc-crystallites becomes smaller. When the surface of sc-crystallites is lower than the certain value, it couldn't act as heterogeneous nucleation sites. This could lead to the decreases of the nucleation efficiency of sc-crystallites. When thermal treatment temperature exceeds $T_{m, s c}$, sc-crystallites are completely destroyed, which would release all PDLA chains. During cooling process, the free PLLA and PDLA chains could form sc-crystallites again. The branched structure of PDLA is beneficial to the alternated arrangement of polymer chains during the formation sc-crystallites. As shown in Fig.6(c), crystallization peaks assigned to sc-crystallites is observed when thermal treatment temperature is higher than $T_{m, s c}$. So the formed sc-crystallites possess the large surface, which meet the requirement of sc-crystallites as heterogeneous nucleation sites [21]. These results reveal that sc-crystallites in the PLLA/L-PDLA blend shows stronger promoting crystallization capacity than the PLLA/6PDLA blend at lower thermal treatment temperature $\left(<T_{m, s c}\right)$. While the re-formed sc-crystallites in the PLLA/6PDLA blends presents the obvious promoting crystallization capacity for PLLA at higher thermal treatment temperature $\left(>T_{m, s c}\right)$ with respect to that of PLLA/L-PDLA. These findings reveal that the promoting crystallization capacity of sc-crystallites for PLLA is 
controlled by thermal treatment temperature and the PDLA structure.

\subsection{Isothermal crystallization behaviors of PLLA/PDLA blends}

Isothermal crystallization kinetics of PLLA/PDLA blends with different structures of PDLA were further studied by DSC at the temperature range $\left(T_{c}=110 \sim 140^{\circ} \mathrm{C}\right)$. Based on the obtained curves of heat flow versus crystallization time (as shown in Fig.S3), crystallization kinetics were analyzed by the Avrami equation $[49,50]$

$$
\log \left[-\ln \left(1-X_{t}\right)\right]=\text { nlogt }+\log \mathrm{k}
$$

where $X_{t}$ is the relative degree of crystallinity; $t$ is crystallization time; $k$ represents the crystallization rate constant; $n$ is the Avrami exponent, which is related to the type of nucleation and the geometry of crystal growth $[48,49]$. As show in Table S1, the $n$ values of the neat PLLA are around 2.46 3.01, revealing a three-dimensional crystallization and homogeneous mechanism. Similar result has been reported by Krikorian et al [51]. For the blends PLLA/L-PDLA and PLLA/4PDLA, the values of $n$ are in the range of 3.39 3.87 (except for $130^{\circ} \mathrm{C}$ ) and 3.26 3.98, suggesting a three-dimensional crystallization growth and heterogeneous nucleation mechanism. For PLLA/3-PDLA, the values of $\mathrm{n}$ are in the range of $1.84 \sim 1.99$ at above $125^{\circ} \mathrm{C}$, while the $n$ values are in the $2.73 \sim 3.03$ at low $120^{\circ} \mathrm{C}$. This reveals that there are two different crystallization mechanisms in the asymmetric PLLA/3PDLA blend, strongly related to the isothermal temperature. At high $T_{c}$, the PLLA of the blend could form the fibrillar crystal, while the flake crystallites could be observed at low $T_{c}$, as reported by Nouri et al[50] For PLLA/6PDLA, the $n$ values is at the range of 
2.45 3.03 except for $T_{c}=110^{\circ} \mathrm{C}$, suggesting a two-dimensional crystallization growth (discoid, flake) and heterogeneous nucleation mechanism. This is attributed to the high cross-linking density of PLLA/6PDLA, limiting the mobility of PLLA chains. The $n$ value of PLLA/6PDLA is 4.11 at $110^{\circ} \mathrm{C}$, indicating a three-dimensional crystallization growth and homogeneous nucleation mechanism as the dominated crystallization mechanism. This is because the lower $T_{c}$ is beneficial to the formation of homogeneous nucleation sites. These results indicate that PLLA/PDLA blends present the different ways of crystal growth due to the different structures of PDLA. The overall crystallization rate constant $k$ is also shown in Table S1. For the neat PLLA, the $k$ values show the decrease trend with increasing of crystallization temperature. This is because the crystallization of the neat PLLA is controlled by homogeneous nucleation mechanism. The low $T_{c}$ is helpful for nucleation sites. However, the $k$ values of the blends don't present the order with increasing of $T_{c}$. This is because the asymmetric PLLA/PDLA blends have a similar branched structure. And rheological results reveal that the cross-linking density of PLLA/PDLA blends is related to the PDLA structure. This makes the blend system more complicated. Meanwhile, the $T_{c}$ affects also crystallization of PLLA. Therefore, this is a common result of the above described factors.

The crystallization half-time $\left(\mathrm{t}_{0.5}\right)$, defined as the time when $X_{t}$ arrives $50 \%$, can obtained from the curves of relative crystallinity versus crystallization time. The obtained $t_{0.5}$ values are shown in Fig. 7(a). As shown in Fig. 7(a), the value of $t_{0.5}$ increases as the $T_{c}$ increases. And the incorporation of PDLA in the PLLA matrix 
obviously decreases the value of $\mathrm{t}_{0.5}$. At lower $T_{c}\left(<130^{\circ} \mathrm{C}\right)$, the $\mathrm{t}_{0.5}$ of the blends decreases in the following order: PLLA/L-PDLA $\approx$ PLLA/4-PDLA $>$ PLLA/3PDLA $\approx$ PLLA/6PDLA, while it becomes lower in the following order: PLLA/L-PDLA $>$ PLLA/4-PDLA $>$ PLLA/3PDLA $>$ PLLA/6PDLA at higher $T_{c}$ $\left(>130^{\circ} \mathrm{C}\right)$. This is closely related to the sc-crystallites reserved in the blends. Therefore, sc-crystallites play two competitive roles: nucleation sites to promote the crystallization rate and cross-linking points to confine the chain mobility. Its nucleation role at low $T_{c}$ is displayed due to the slow mobility of polymer chain. At high $T_{c}$, the mobility of polymer chains is accelerated, leading to the full display of the formed network cross-linking effect. Consequently, this indicates that the PDLA structure has the obvious effect on the PLLA crystallization due to the difference of sc-crystallites formed by linear PLLA and PDLA with different structures.

Fig. 7(b) shows the crystallinity of blends after isotherm-crystalized at different temperature. For PLLA/L-PDLA, its crystallinity first increases and then decreases with increasing of $T_{c}$. At higher $T_{c}$, polymer chains show good movement capacity, which is advantage for PLLA crystallization. With decreasing of $T_{c}$, the movement capacity of polymer chains decreases, resulting in the decreasing of crystallinity. When the $T_{c}$ further decreases, homogeneous nucleation is the dominated mechanism. This would promote PLLA crystallization again. However, the crystallinity of PLLA/S-PDLA is always increases with increasing of $T_{c}$. In the blends PLLA/S-PDLA, there have larger cross-linking points than PLLA/L-PDLA. This is 
because the branched point of S-PDLA may also play a role of cross-linking point. So the crystallization of PLLA/S-PDLA is mainly controlled by the movement of polymer chain. With increasing of $T_{c}$, the movement capacity of polymer chain increases, which would promote crystallization. It can be found that the crystallinity of PLLA/S-PDLA presents the trend: PLLA/4PDLA>PLLA/3PDLA>PLLA/6PDLA. At present the interpretation for this phenomenon subjects to further discussion. It may be related to nucleation efficiency and cross-linking effect of sc-crystallite, and the PDLA structure.

Melting temperature of polymer is closely related to crystallite structure, so polymorphic structure of PLLA/PDLA blends isotherm-crystallized at different temperatures can be evaluated from the melting behavior. Fig. 8 shows the DSC melting curves of PLLA/PDLA blends isotherm-crystallized at different temperatures. It can be found that the blends isotherm-crystallized at $110^{\circ} \mathrm{C}$ show double melting peaks, which may be attributed to the melt recrystallization mechanism. With increasing of $T_{c}$, the double melting peaks merge into one peak and the temperature corresponded to the melting peak increases. At lower $T_{c}$, PLLA could form homogeneous nucleation sites due to the low mobility capacity of PLLA chain. Therefore, there are both homogeneous- and heterogeneous nucleation, which leads to different perfect degree of crystallites. At higher $T_{c}$, there has only heterogeneous nucleation because the mobility of PLLA chain accelerates, improving the perfect degree of the crystallites. So there does not undergo recrystallization upon heating. Similar results have been reported by Pan [52]. It is also clear that the PDLA structure 
doesn't affect the crystal structure.

\subsection{Nucleation and spherulite growth}

To investigate the nucleation effect of sc-crystallites in the PLLA/PDLA blends for PLLA, the nucleation efficiency of sc-crystallites was employed. A nucleation efficiency scale for a given polymer is determined by cooling a polymer sample from the melt. Upon cooling, the sample will crystallize at a given temperature, and an effective nucleating agent will cause the crystallization temperature $\left(T_{c}\right)$ of the homopolymer to increase, with a higher temperature corresponding to an increased level of nucleation. The nucleation efficiency can be calculated by the followed equation [32]

$$
\mathrm{NE}(\%)=\frac{T_{c}-T_{\min }}{T_{\max }-T_{\min }} \times 100 \%
$$

where $T_{\max }$ represents the maximum crystallization temperature of polymer after self-nucleation; $T_{\min }$ is the crystallization temperature of pure polymer; $T_{\mathrm{c}}$, which can be acquired from Fig.5(a), is the crystallization temperature at cooling process. Self-nucleation is considered to be the ideal case for pure polymer crystallization due to an optimum dispersion of crystallites and the favorable interaction between polymer melt and polymer crystal fragment. Fig. 9(a) shows DSC cooling curves of the neat PLLA after self-nucleation at different temperature. It is obvious that crystallization temperature at the self-nucleation temperature of $190^{\circ} \mathrm{C}$ is the minimum $\left(T_{\min }=100.9^{\circ} \mathrm{C}\right)$. With decreasing of self-nucleation temperature, crystallization temperature obviously increases. This is because the decreased temperature would occur the partial melting zone, where exists a large number of 
self-nucleation seeds. $T_{\max }$ should appear at the lowest self-nucleation temperature of the partial melting zone which is defined to the temperature at which stable crystal fragments arrive at the saturated sate and insufficient melting occurs at temperatures when self-nucleation temperature is lower than it. As show in Fig. 9(b), the melting peak shifts to low temperature disappears as self-nucleation temperature increases, and the melting peak disappears at self-nucleation temperature higher than $168^{\circ} \mathrm{C}$. This indicates that $168^{\circ} \mathrm{C}$ is the lowest self-nucleation temperature of the partial melting zone. Therefore, $T_{\max }=132.2^{\circ} \mathrm{C}$ could be acquired.

Fig. 10 shows nucleation efficiency of sc-crystallites in the PLLA/PDLA blends to PLLA during non-isothermal crystallization. For the blends PLLA/L-PDLA, its nucleation efficiency could arrive at $66.1 \%$. It is clear that the nucleation efficiency decreases with increasing of arm number of PDLA added in the PLLA/PDLA blends. Even the nucleation efficiency of sc-crystallites in the blends PLLA/6PDLA is the negative value $(-13.4 \%)$. This may be closely related to the structure of PDLA. Sc-crystallites could be formed by the strong hydrogen bonding between PLLA segment and PDLA segment. So in the process of sc-crystallite formation, the number of PLLA segment is incorporated into sc-crystallites. This may be form an analogous star-sharp structure, where sc-crystallites acts as the nuclear and the surplus PLLA segment acts as arm. While with increasing of arm number of PDLA, the arm length of PDLA with similar molecular weight becomes short. This may form smaller sc-crystallites that haven't the enough surface area to act as nucleation sites. Furthermore, sc-crystallites could confine the movement of PLLA segment due to its 
cross-linking structure, decreasing its nucleation efficiency. Fig.11 show spherulite morphology of PLLA and PLLA/PDLA blends during isothermal crystallization of $130^{\circ} \mathrm{C}$ after cooling $190^{\circ} \mathrm{C}$. For PLLA/PDLA blends, the density of spherulites increases with the addition of PDLA with respect to the neat PLLA. These may be attributed to heterogeneous nucleation of sc-crystallites reserved in the PLLA melt. The spherulite density decreases in the following order: 4PDLA/PLLA>6PDLA/PLLA>L-PDLA/PLLA>3PDLA/PLLA. This may be attributed to the stereocomplex network structure formed at asymmetric PLLA/PDLA blends, which results in the two roles of sc-crystallites: nucleation sites and cross-linking points, as proved by rheological results and DSC results.

\section{Conclusions}

This study has found that the asymmetric PLLA/PDLA blends exists the complicated network structure, which is closely related to sc-crystallites and the branched structure of PDLA. Rheology indicated that sc-crystallite reserved in the melt is a good rheological modifier, and could achieve the transition from the liquid-like to solid-like viscoelatic behavior. Effect of PDLA on rheological behavior of the melt complies with the sequence: 6PDLA>L-PDLA>3PDLA>4PDLA. Dissolution experiment revealed that the sc-crystallites network in the melt of PLLA could be formed by interparticle polymer chains which are restrained by the cross-linking effect of sc-crystallites or branch points of PDLA. Crystallization and melting behaviors of the blends were deeply analyzed by DSC, indicating that the crystallization capacity of the blends is closely related to thermal treatment 
temperature and the structure of PDLA. PDLA promotes obviously the PLLA crystallization when cooled from $190^{\circ} \mathrm{C}$, and the promoting crystallinity capacity complies with the sequence: 4PDLA $>$ L-PDLA $>6$ PDLA $>3$ PDLA, which is attributed to sc-crystallites in the PLLA/PDLA blends. Sc-crystallites in the PLLA/L-PDLA blend shows stronger promoting crystallization capacity than the PLLA/6PDLA blend at lower thermal treatment temperature $\left(<T_{m, s c}\right)$. While the re-formed sc-crystallites in the PLLA/6PDLA blends presents the obvious promoting crystallization capacity for PLLA at higher thermal treatment temperature $\left(>T_{m, s c}\right)$ with respect to that of PLLA/L-PDLA. Isothermal crystallization revealed that the reserved sc-crystallites in the PLLA/PDLA blends presents different the promoting crystallization capacity due to the different PDLA structure. And the nucleation efficiency of sc-crystallites in the blends decreases with increasing of the arm numbers of PDLA. POM results reveal that spherulite density decreases in the following order: 4PDLA/PLLA>6PDLA/PLLA>L-PDLA/PLLA>3PDLA/PLLA.

\section{Acknowledgements}

The authors thank for jointly supporting by the National Science Foundation of China (No. 51303149), foundation for the Fundamental Research Funds for Central Universities (3102014JC01095).

\section{References}

[1] Rasal RM, Janorkar AV, Hirt DE. Prog Polym Sci 2010; 35: 338-56.

[2] Saeidlou S, Huneault MA, Li H, Park CB. Prog Polym Sci 2012; 37: 1657-77.

[3] Shao J, Sun J, Biao X, Cui Y, Li G, Chen X. J Phys Chem B 2012; 116: 9983-91. 
[4] Mathew AP, Oksman K, Sain M. J Appl Polym Sci 2005; 97: 2014-25.

[5] Pei A, Zhou Q, Berglund L A. Compos Sci Technol 2010; 70: 815-21.

[6] Jing Z, Shi X, Zhang G, Qin J. Polym Adv Technol 2015; 26: 223-33.

[7] Hashima K, Nishitsuji S, Inoue T. Polymer 2010; 51: 3934-39.

[8] Wang L, Jing X, Cheng H, Jing X, Cheng H, Hu X, et al. Ind Eng Chem Res 2012; 51: 10731-41.

[9] Nampoothiri KM, Nair NR, John RP. Bioresour Technol 2010; 101: 8493-501.

[10] Martin O, Avérous L. Polymer 2001; 42: 6209-12.

[11] Bai H, Zhang W, Deng H, Zhang Q, Fu Q. Macromolecules 2011; 44: 1233-7.

[12] Zeng X, Wu B, Wu L, Hu J, Bu Z, Li BG. Ind Eng Chem Res 2014; 53: 3550-8.

[13] Hirata M, Masutani K, Kimura Y. Biomacromolecules 2013; 14: 2154-61.

[14] Ikada Y, Jamshidi K, Tsuji H, Hyon SH. Macromolecules 1987; 20: 904-906.

[15] Sun Y, He C. ACS Macro Lett 2012; 1: 709-13.

[16] Zhang P, Tian R, Na B, Lv R, Liu Q. Polymer 2015; 60: 221-7.

[17] Shi WP, Zhao CY, Li SM, Fan ZY. Chem J Chinese U 2012; 33: 2092-98.

[18] Tsuji H, Miyase T, Tezuka Y, Saha SK. Biomacromolecules 2005: 6: 244-54.

[19] Tsuji H, Yamashita Y. Polymer 2014, 55: 6444-50.

[20] Song Y, Zhang X, Yin Y, Vos S de, Wang R, Joziasse C A P, et al. Polymer 2015, 72: 185-92.

[21] Yamane H, Sasai K. Polymer 2003; 44: 2569-75.

[22] Ma P, Shen T, Xu P, Dong W, Lemstra PJ, Chen M. ACS Sustainable Chem Eng 2015; 3: 1470-78. 
[23] Wei XF, Bao RY, Cao ZQ, Yang W, Xie BH, Yang MB. Macromolecules 2014; 47: 1439-48.

[24] Tsuji H, Takai H, Saha SK. Polymer 2006; 47: 3826-37.

[25] Xu H, Tang S, Chen J, Chen N. Polym-Plast Technol 2015; 54: 1-13.

[26] Xu H, Tang S, Chen J. Polym-Plast Technol 2013; 52: 690-8.

[27] Rahman N, Kawai T, Matsuba G, Nishida K, Kanaya T, Watanabe H, Okamoto H, et al. Macromolecules 2009; 42: 4739-45.

[28] Wen T, Xiong Z, Liu G, Zhang X, Vos S de, Wang R, et al. Polymer 2013; 54: 1923-9.

[29] Urayama H, Kanamori T, Fukushima K, Kimura Y. Polymer 2003; 44: 5635-41.

[30] Brochu S, Prud'Homme RE, Barakat I, Jerome R. Macromolecules 1995; 28: 5230-9.

[31] Xiong Z, Zhang X, Wang R, Vos S de, Wang R, Joziasse C A P, et al. Polymer 2015; 76: 98-104.

[32] Anderson KS, Hillmyer MA. Polymer 2006; 47: 2030-35.

[33] Jing Z, Shi X, Zhang G, Li J. Polym Adv Technol 2015; 26: 528-37.

[34] Xiong Z, Liu G, Zhang X, Wen T, Vosc S de, Joziassec C, Wang D. Polymer 2013; 54: 964-971.

[35] Sun Y, He C. RSC Adv 2013; 3: 2219-26.

[36] Jing Z, Shi X, Zhang G, Lei R. Polym Int 2015; 64: 1399-407.

[37] Xu Z, Zhang Y, Wang Z, Sun N, Li H. ACS Appl Mater Interfaces 2011; 3: 4858-64. 
[38] Liu K, Andablo-Reyes E, Patil N, Merino D H, Ronca S, Rastogi S, Polymer 2016; 87: 8-16.

[39] Xu Z, Niu Y, Yang L, Xie W, Li H, Gan Z, Wang Z. Polymer 2010; 51: 730-7.

[40] Wood-Adams PM, Dealy JM, deGroot AW, Redwine OD. Macromolecules 2000: 33: 7489-99.

[41] Yamane H, Sasai K, Takano M, Takahashi, M. J Rheol 2004; 48: 599-609.

[42] Acierno S, Grizzuti N. J Rheol 2003; 47: 563-76.

[43] Fujita M, Sawayanagi T, Abe H, Tanaka T, Iwata T, Ito K, et al. Macromolecules 2008; 41: 2852-2858.

[44] Brizzolara D, Cantow HJ, Diederichs K, Keller E, Domb AJ. Macromolecules 1996; 29: 191-7.

[45] Horst RH, Winter HH. Macromolecules 2000; 33: 7538-43.

[46] Coppola S, Acierno S, Grizzuti N, Vlassopoulos D. Macromolecules 2006; 39: 1507-14.

[47] Li Y, Xin S, Bian Y, Dong Q, Han C, Xu K, et al. RSC Adv 2015; 5: 24352-62.

[48] Narita J, Katagiri M, Tsuji H. Macromol Mater Eng 2013; 298: 270-82.

[49] Han L, Pan P, Shan G, Bao Y. Polymer 2015; 63: 144-53.

[50] Nouri S, Dubois C, Lafleur PG. Polymer 2015; 67: 227-39.

[51] Krikorian V, Pochan D J. Macromolecules 2004; 37: 6480-91.

[52] Pan P, Han L, Bao J, Xie Q, Shan G, Bao Y. J Phys Chem B 2015; 119: 6462-70. 


\section{Figures and Tables Caption}

Table 1 Synthesis and characterization of PDLA with different structures

Table 2 Thermal parameters of PLLA and PLLA/PDLA blends obtained from DSC

Fig. 1. (a) the finger print region of $1000-850 \mathrm{~cm}^{-1}$ at FT-IR spectra of PLLA/PDLA blends; (b) DSC curves of PLLA/PDLA blends at the first heating scan; (c) XRD curves of PLLA/PDLA blends

Fig. 2. Variation of (a) storage modulus $\left(G^{\prime}\right)$, (b) loss modulus $\left(G^{\prime \prime}\right)$, (c) loss tangent $(\tan \delta)$, and (d) complex viscosity $\left(\eta^{*}\right)$ as functions of frequency for PLLA/PDLA blends

Fig. 4. Schematic diagrams for the probable structures of the formed network in the blends PLLA/L-PDLA (a) and PLLA/4PDLA (b)

Fig. 3. The size distribution of PLA stereocomplex dispersed in $\mathrm{CH}_{2} \mathrm{Cl}_{2}$ determined by DLS

Fig. 5. DSC cooling curves by $2^{\circ} \mathrm{C} / \mathrm{min}$ (a) and heating curves by $10^{\circ} \mathrm{C} / \mathrm{min}$ (b) of PLLA/PDLA blends after melting at $190^{\circ} \mathrm{C}$

Fig. 6. DSC cooling curves by $2^{\circ} \mathrm{C} / \mathrm{min}(\mathrm{a}, \mathrm{c})$ and heating curves by $10^{\circ} \mathrm{C} / \mathrm{min}(\mathrm{b}, \mathrm{d})$ of PLLA/L-PDLA and PLLA/6PDLA after melting for 4 min at different temperatures Fig. 7. Crystallization half-time (a) and crystallinity (b) of PLLA/PDLA blends isotherm-crystallized at different temperatures

Fig. 8. DSC melting curves of the blends isotherm-crystallized at different temperatures: (a) PLLA/L-PDLA, (b) PLLA/3PDLA, (c) PLLA/4PDLA, (d) PLLA/6PDLA 
Fig. 9. (a) DSC cooling curves of the neat PLLA after self-nucleation at different temperatures; (b) DSC heating curves of samples after being annealed at different temperatures for 4 min

Fig. 10. Nucleation efficiency (NE) of sc-crystallites formed PLLA and PDLA with different structures to PLLA

Fig. 11. Spherulite morphologies of PLLA/PDLA blends during isothermal crystallization of $130^{\circ} \mathrm{C}$ after cooling from $190^{\circ} \mathrm{C}$ 
Table 1 Synthesis and characterization of PDLA with different structures

\begin{tabular}{lccccccc}
\hline Samples & $\begin{array}{c}\text { [D-lactide]/[initiator }] \\
(\mathrm{mol} / \mathrm{mol})\end{array}$ & $\begin{array}{c}M_{n}{ }^{a} \\
\left(\times 10^{4} \mathrm{~g} / \mathrm{mol}\right)\end{array}$ & $\begin{array}{c}M_{w}{ }^{a} \\
\left(\times 10^{4} \mathrm{~g} / \mathrm{mol}\right)\end{array}$ & $\mathrm{PDI}^{\mathrm{a}}$ & $T_{c}{ }^{b}$ & $T_{m}{ }^{b}$ & Yeild $(\%)$ \\
\hline L-PDLA & 150 & 2.76 & 4.75 & 1.72 & 139.2 & 170.6 & 88.1 \\
3PDLA & 150 & 2.65 & 5.08 & 1.91 & 129.7 & 164.2 & 74.2 \\
4PDLA & 150 & 3.47 & 6.46 & 1.86 & 103.1 & 164.9 & 90.2 \\
6PDLA & 150 & 3.51 & 6.68 & 1.90 & 100.2 & $163.7 / 151.9$ & 80.9 \\
\hline
\end{tabular}

${ }^{\mathrm{a}}$ Determined by GPC.

${ }^{\mathrm{b}}$ Determined by DSC. 
Table 2 Thermal parameters of PLLA and PLLA/PDLA blends obtained from DSC

\begin{tabular}{ccccccc}
\hline Samples & $T_{c}\left({ }^{\circ} \mathrm{C}\right)$ & $T_{c c}\left({ }^{\circ} \mathrm{C}\right)$ & $T_{m}\left({ }^{\circ} \mathrm{C}\right)$ & $\Delta H_{c c}(\mathrm{~J} / \mathrm{g})$ & $\Delta H_{m}(\mathrm{~J} / \mathrm{g})$ & $X(\%)$ \\
\hline PLLA & 100.9 & 114.1 & $162.9 / 167.4$ & 19.0 & 33.4 & 15.5 \\
PLLA/L-PDLA & 121.6 & -- & 165.4 & $-\ldots$ & 28.9 & 32.7 \\
PLLA/3PDLA & 118.1 & 105.4 & 166.1 & 4.8 & 24.4 & 22.2 \\
PLLA/4PDLA & 115.4 & -- & 164.7 & -- & 30.3 & 34.3 \\
PLLA/6PDLA & 96.7 & 98.4 & 116.1 & 2.2 & 25.5 & 26.4 \\
\hline
\end{tabular}

- -Not detected.

DSC programs: cooling from $190^{\circ} \mathrm{C}$ by $2{ }^{\circ} \mathrm{C} / \mathrm{min}$ and then heating to $190^{\circ} \mathrm{C}$ by $10^{\circ} \mathrm{C} / \mathrm{min}$. 

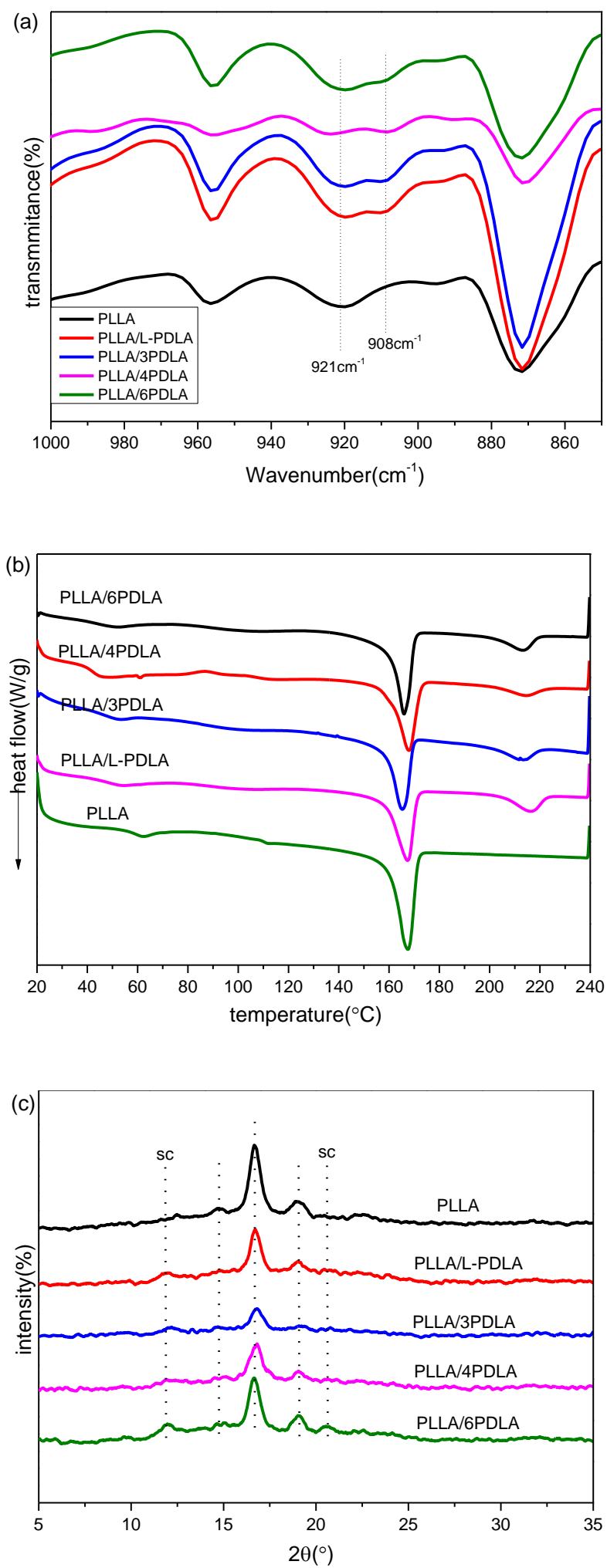

Fig. 1. (a) the finger print region of $1000-850 \mathrm{~cm}^{-1}$ at FT-IR spectra of PLLA/PDLA blends; (b) DSC curves of PLLA/PDLA blends at the first heating scan; (c) XRD curves of PLLA/PDLA blend 

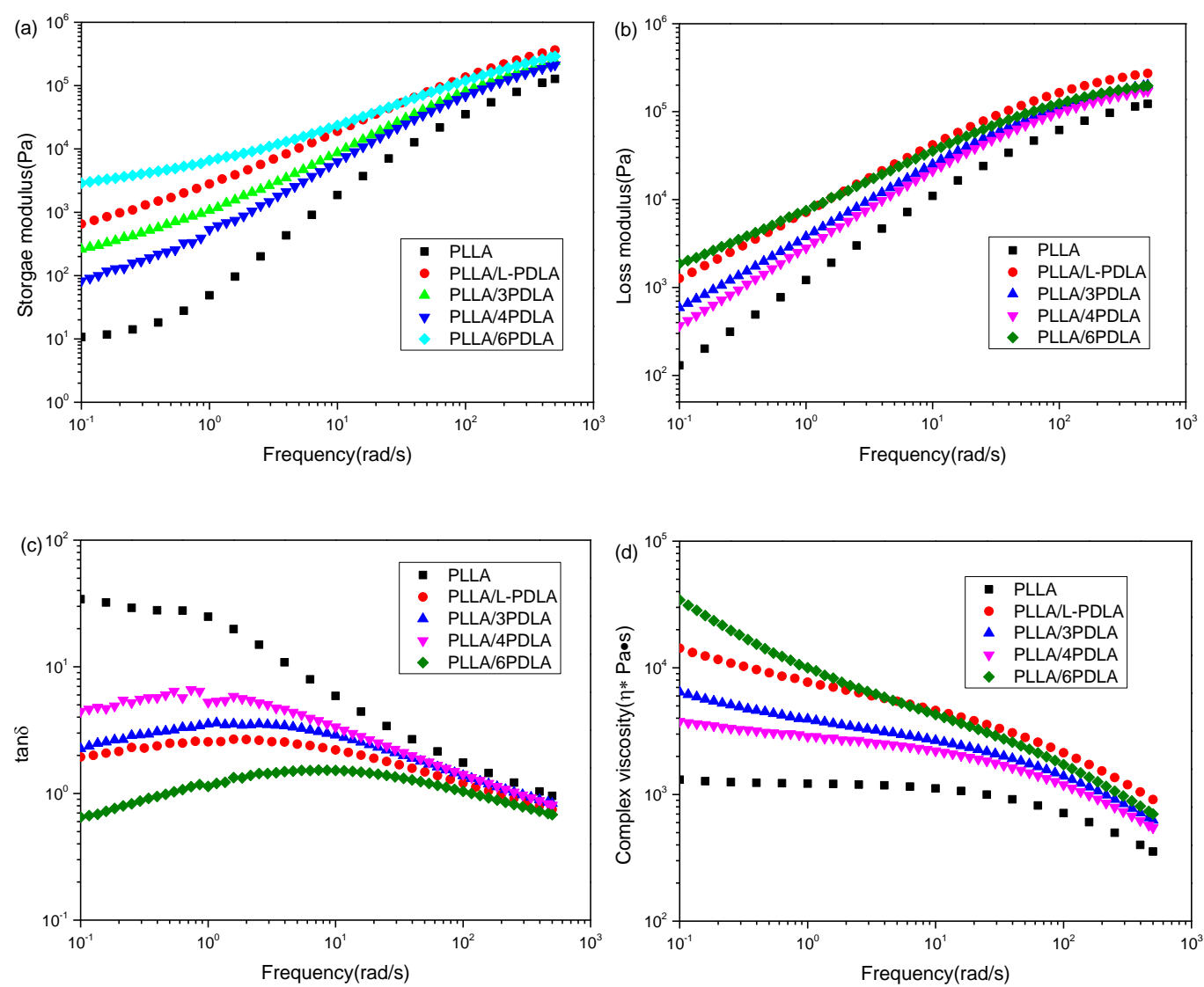

Fig. 2. Variation of (a) storage modulus $\left(G^{\prime}\right)$, (b) loss modulus $\left(G^{\prime \prime}\right)$, (c) loss tangent $(\tan \delta)$, and (d) complex viscosity $\left(\eta^{*}\right)$ as functions of frequency for PLLA/PDLA blends 


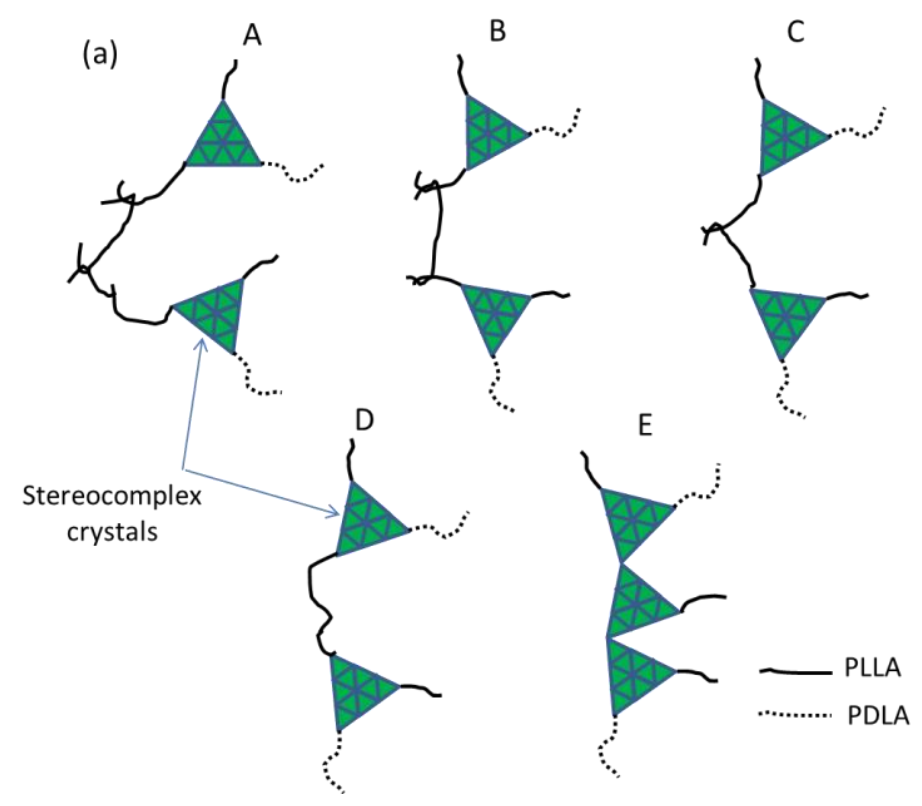

(b)
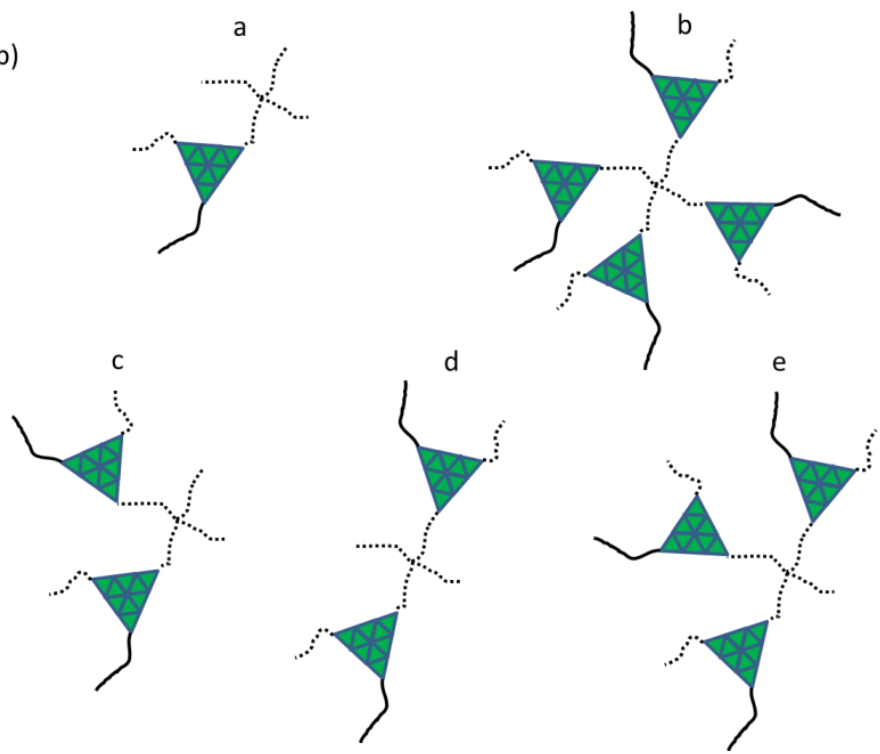

Fig. 3. Schematic diagrams for the probable structures of the formed network in the blends PLLA/L-PDLA (a) and PLLA/4PDLA (b) 

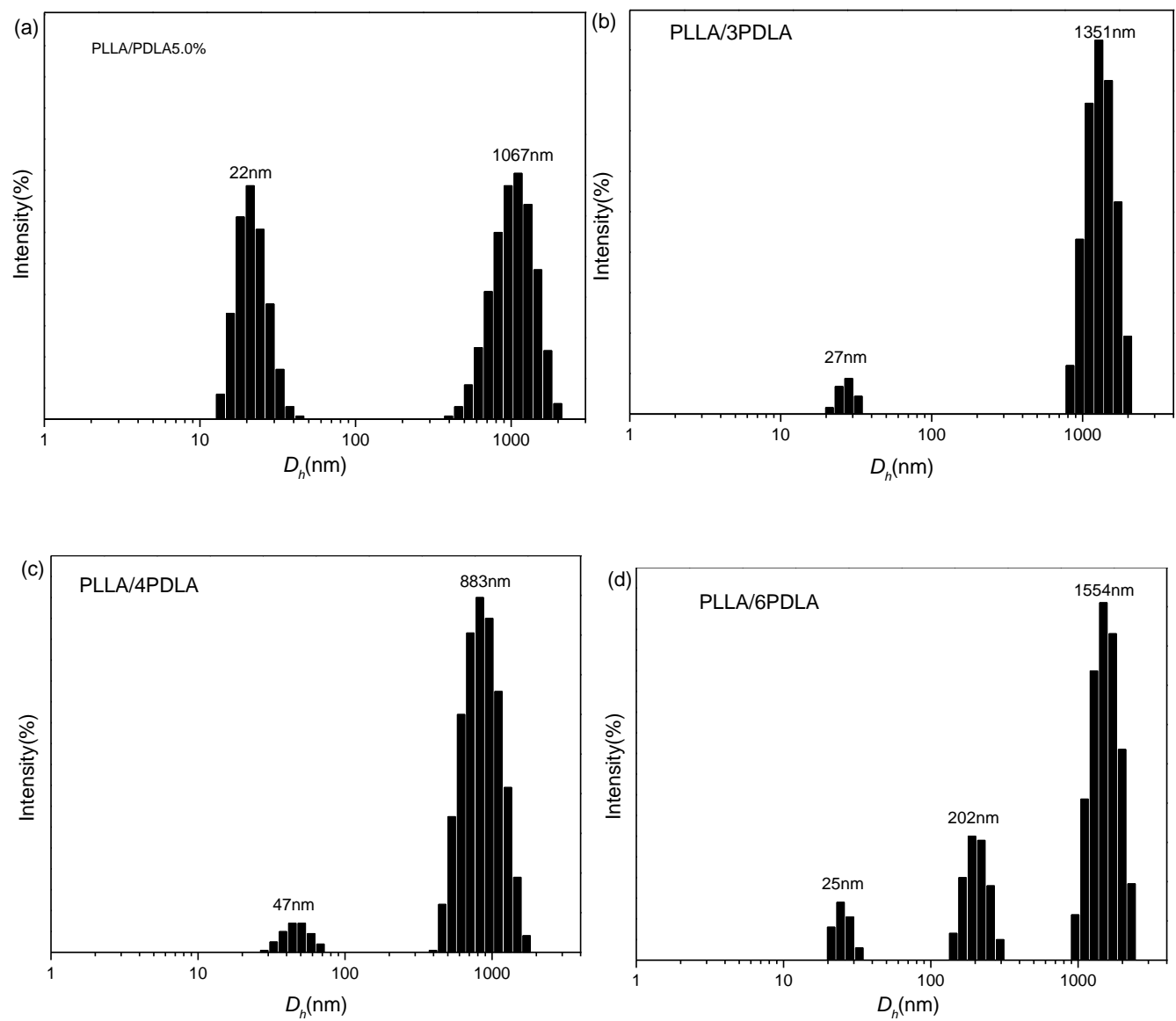

Fig. 4. The size distribution of PLA stereocomplex dispersed in $\mathrm{CH}_{2} \mathrm{Cl}_{2}$ determined by DLS 


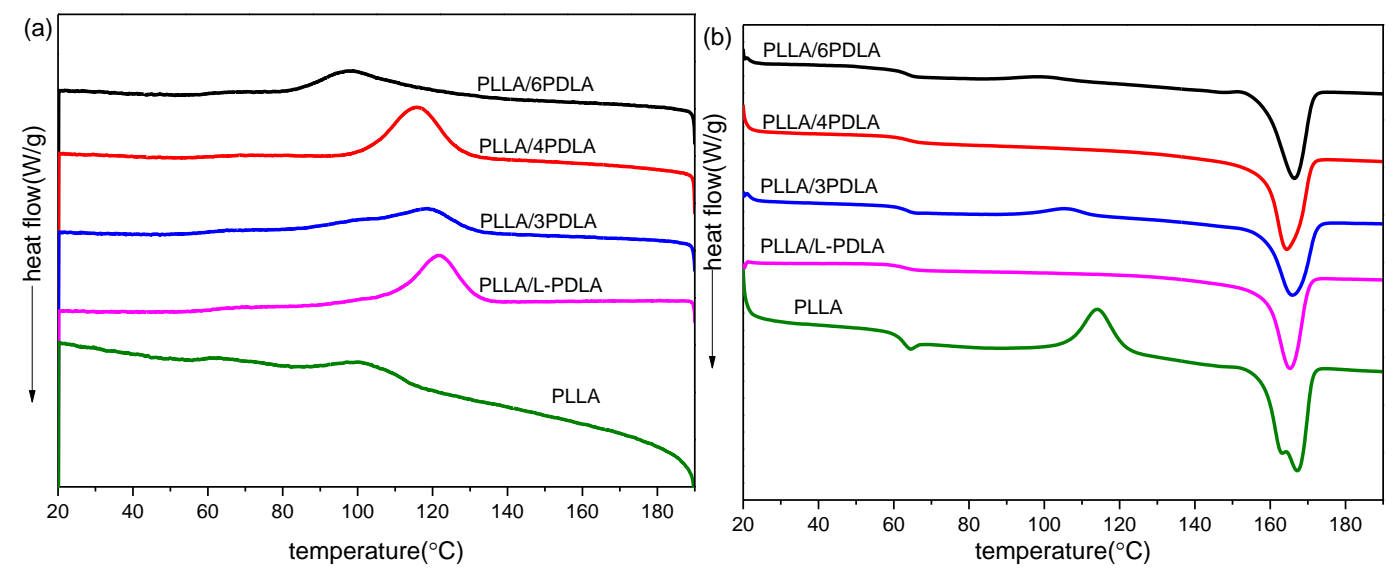

Fig. 5. DSC cooling curves by $2^{\circ} \mathrm{C} / \mathrm{min}$ (a) and heating curves by $10^{\circ} \mathrm{C} / \mathrm{min}$ (b) of PLLA/PDLA blends after melting at $190^{\circ} \mathrm{C}$ 

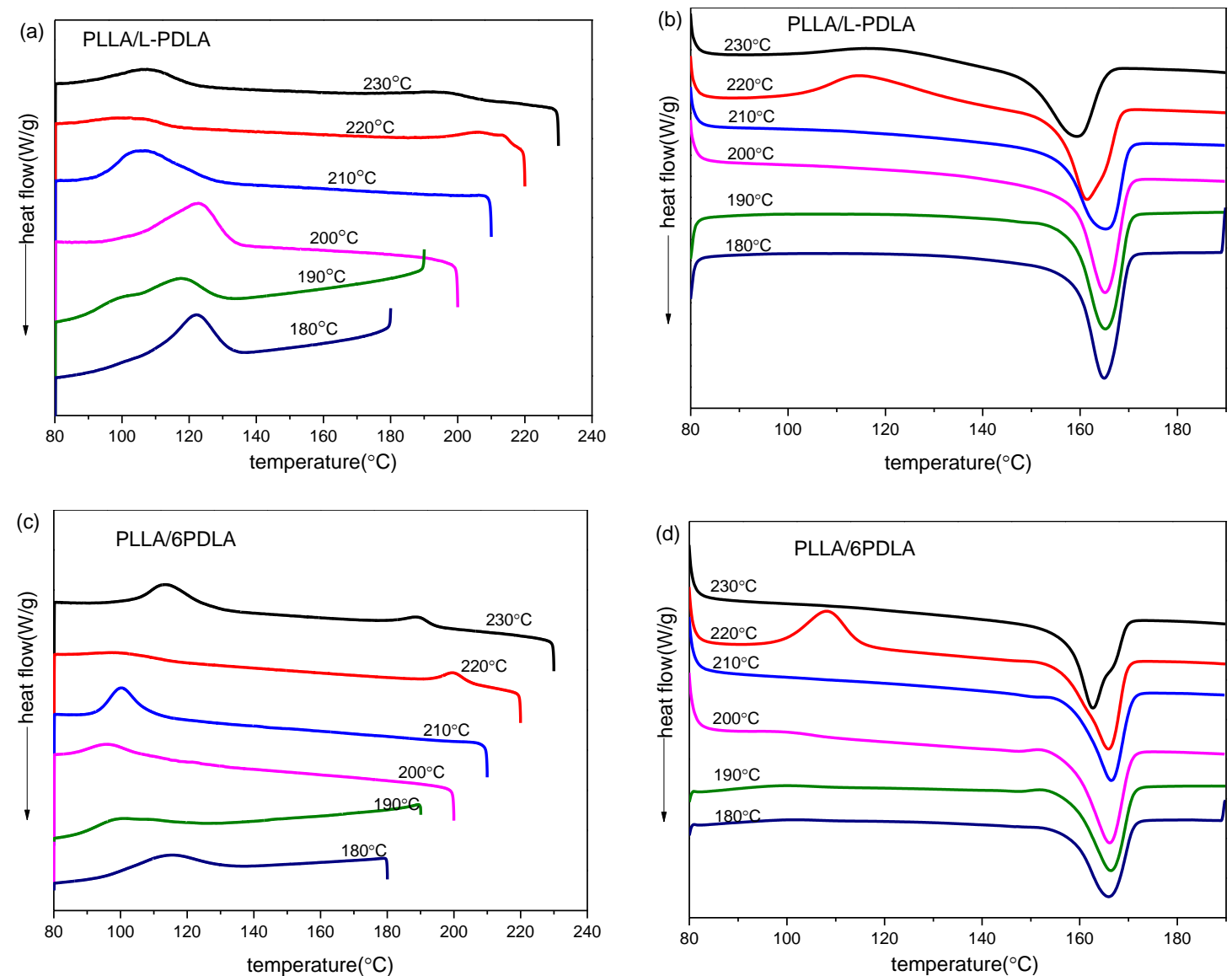

Fig. 6. DSC cooling curves by $2{ }^{\circ} \mathrm{C} / \min (\mathrm{a}, \mathrm{c})$ and heating curves by $10{ }^{\circ} \mathrm{C} / \mathrm{min}(\mathrm{b}, \mathrm{d})$ of PLLA/L-PDLA and PLLA/6PDLA after melting for $4 \mathrm{~min}$ at different temperatures 

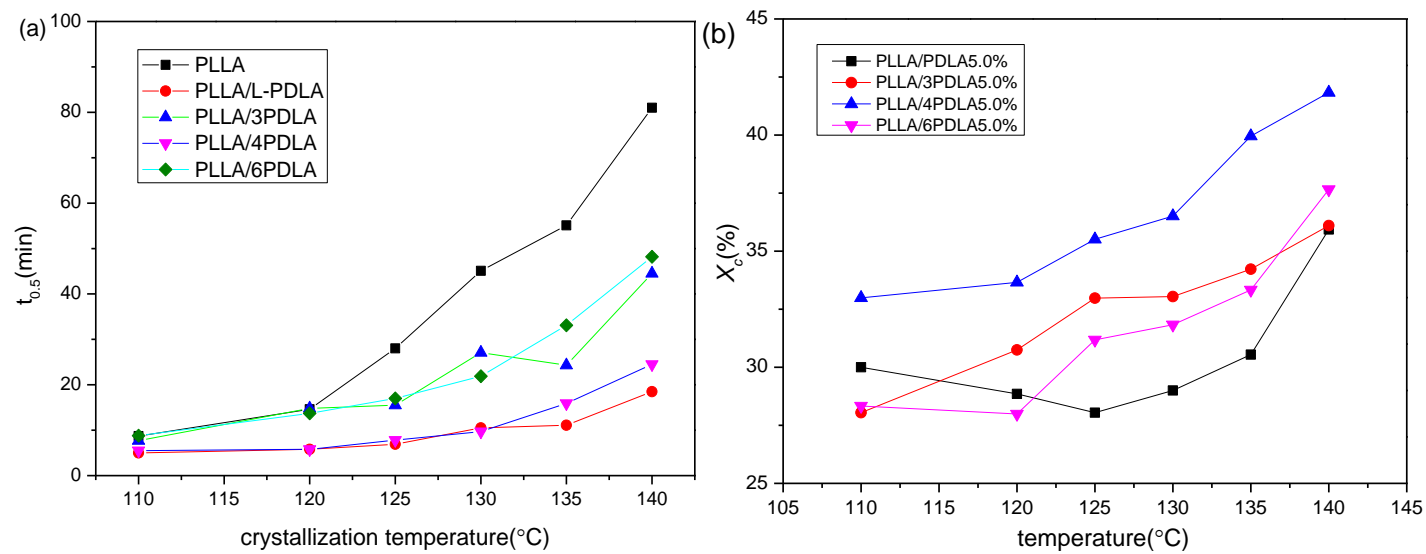

Fig. 7. Crystallization half-time (a) and crystallinity (b) of PLLA/PDLA blends isotherm-crystallized at different temperatures 

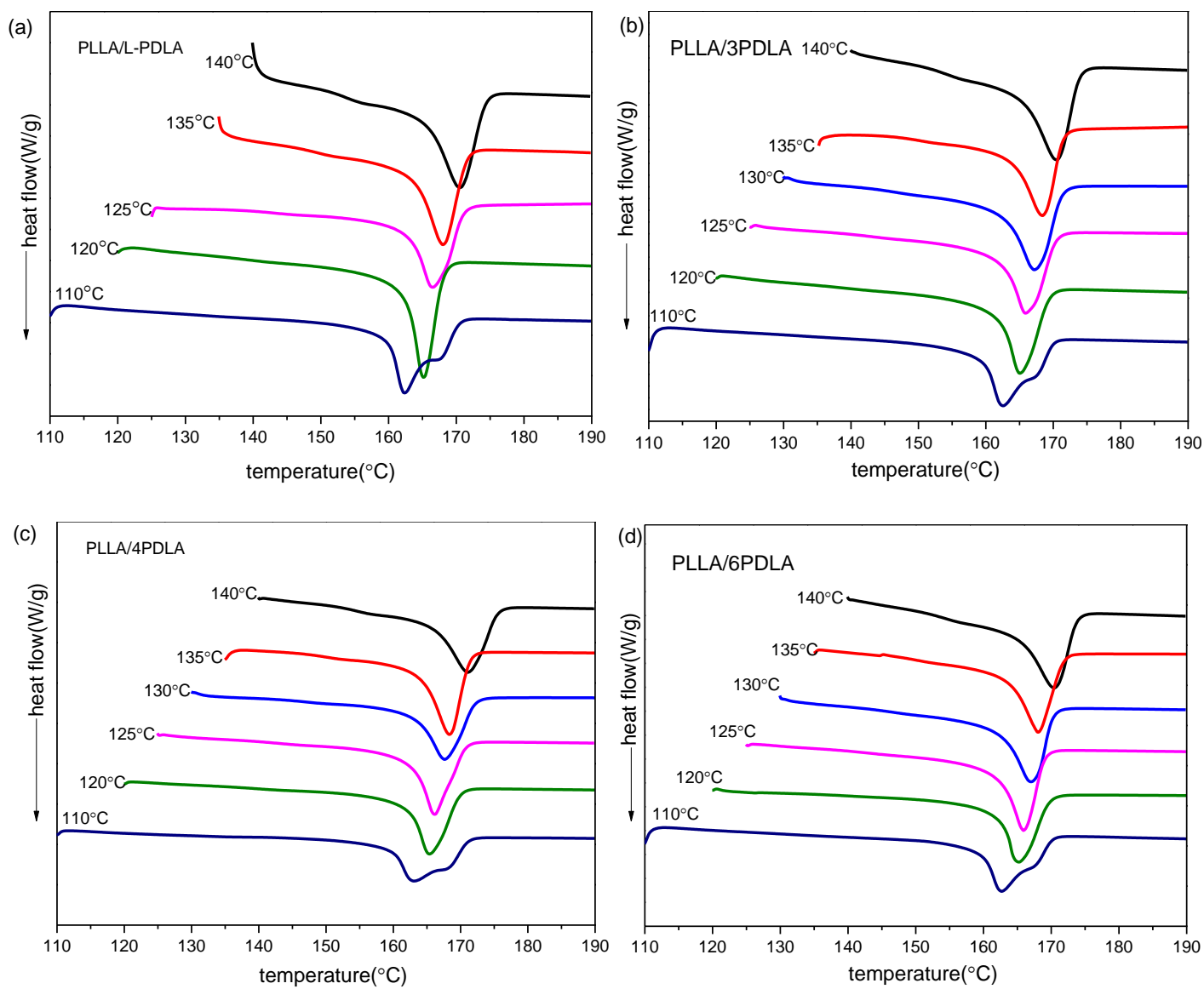

Fig. 8. DSC melting curves of blends isotherm-crystallized at different temperatures:

(a) PLLA/L-PDLA, (b) PLLA/3PDLA, (c) PLLA/4PDLA, (d) PLLA/6PDLA 

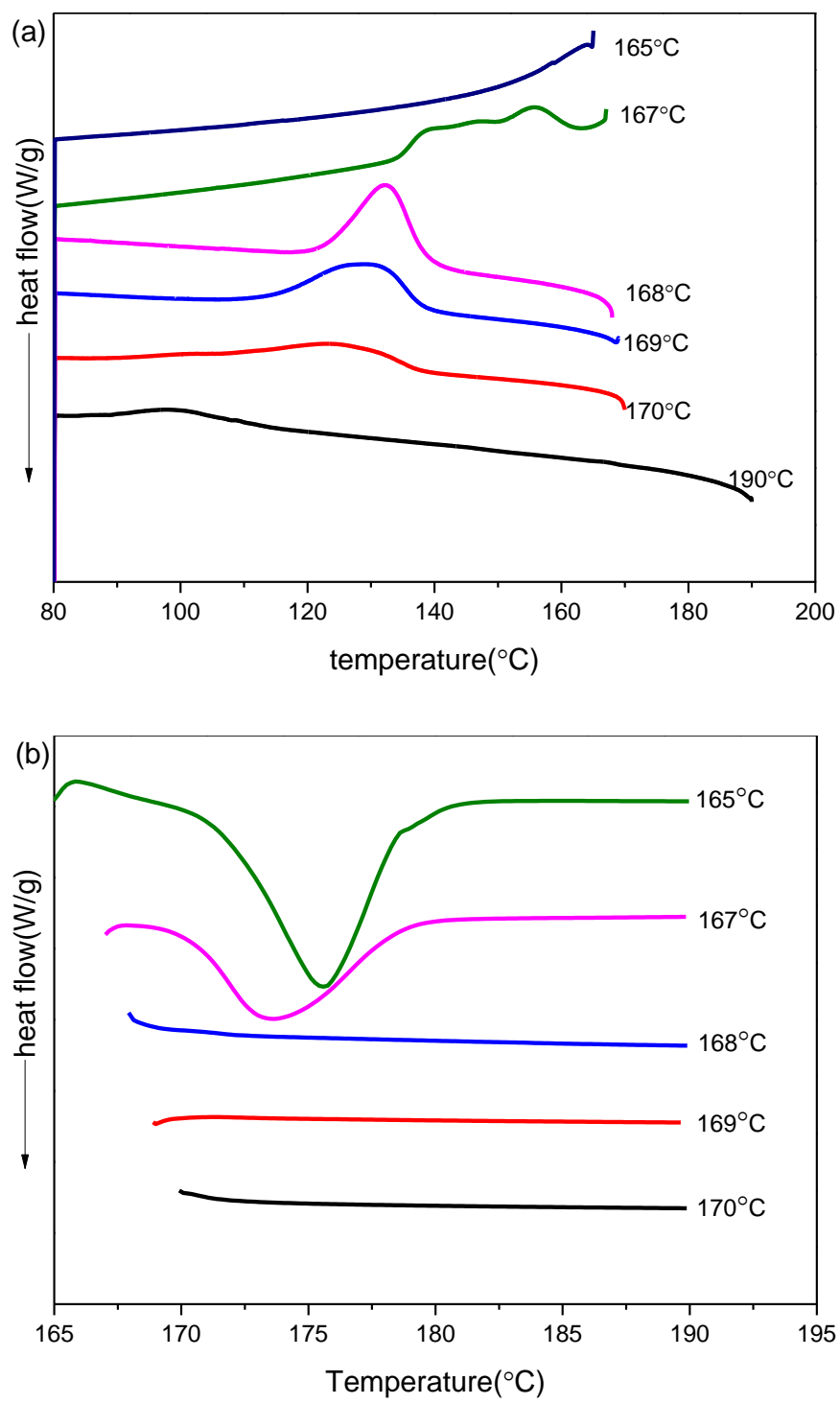

Fig. 9. (a) DSC cooling curves of the neat PLLA after self-nucleation at different temperatures; (b) DSC heating curves of samples after being annealed at different temperatures for $4 \mathrm{~min}$ 


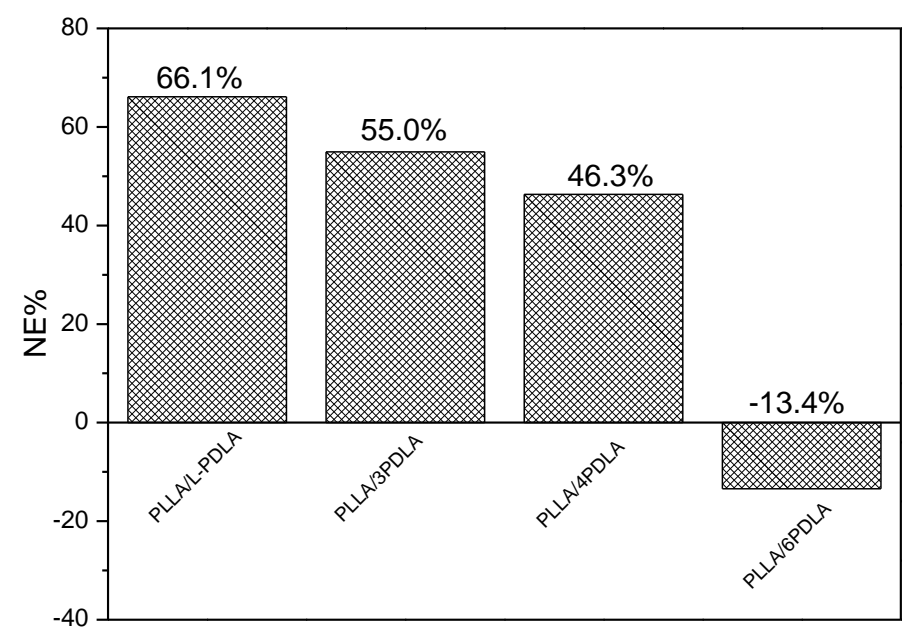

Fig. 10. Nucleation efficiency (NE) of sc-crystallites formed PLLA and PDLA with different structures to PLLA 


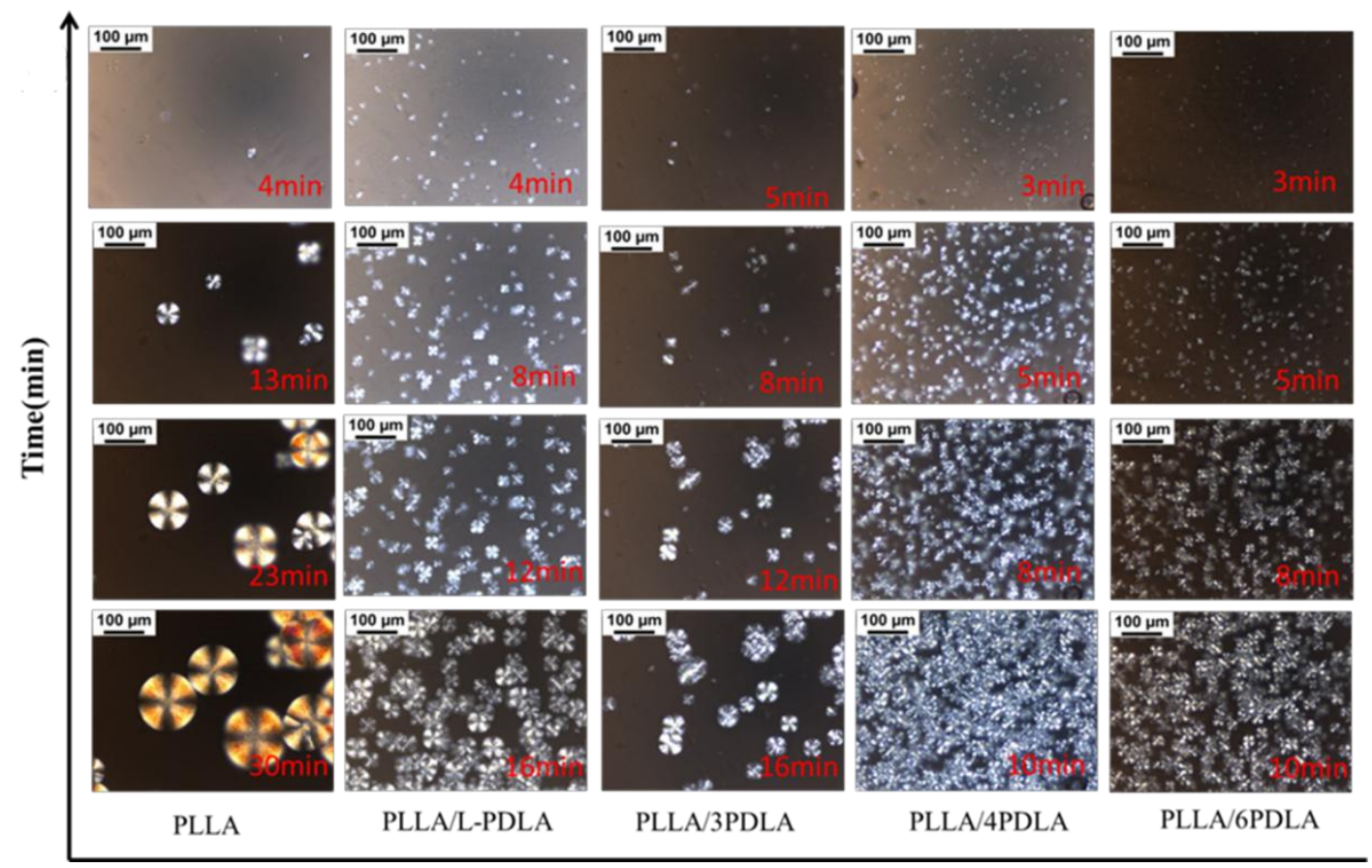

Fig. 11. Spherulite morphologies of PLLA/PDLA blends during isothermal crystallization of $130^{\circ} \mathrm{C}$ after cooling from $190^{\circ} \mathrm{C}$ 


\section{Graphical Abstract}
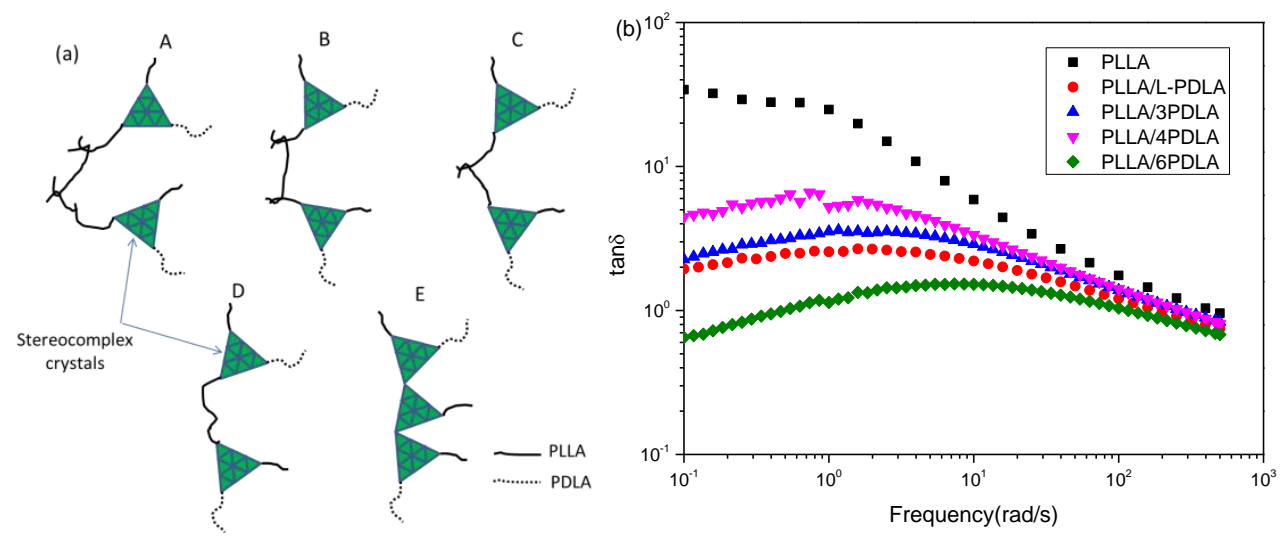